\title{
Long-term Follow-up (Minimum 5 Years) Study of Single-level Posterior Dynamic Stabilization in Lumbar Degenerative Disease; 'Interspinous U' \& 'DIAM'
}

\author{
Yeon Joon Kim, Sang Gu Lee, Chan Woo Park, Seong Son, Woo Kyung Kim \\ Department of Neurosurgery, Gachon University, Gil Hospital, Incheon, Korea
}

Objectives: Recently posterior dynamic stabilizations (PDS) are increased in degenerative lumbar disease. But, some previous studies had doubts its long term prognosis. Long term clinical and radiological results of PDS using interspinous device (Interspinous U, DIAM) were analyzed.

Methods: We have used the 'interspinous U' and 'DIAM' for patients with lumbar spinal stenosis. We included single level lumbar spinal stenosis patients who completed minimum 60 months follow-up evaluation. All patients checked plain lateral and flexion-extension views at immediately after the surgery and each follow-up. The clinical outcome was measured by Odom's criteria. Complications including post operative infection, bony erosion, device fracture, device malformations, and instabilities were surveyed.

Results: We included 18 for 'Interspinous U' and 7 patients 'DIAM' groups. Mean follow-up durations for 'Interspinous U' and 'DIAM' were 74.6 and 62.6 months, respectively. Satisfactory groups were $50.0 \%$ and $42.9 \%$ for 'Interspinous U' and 'DIAM' groups. In 'Interspinous U' group disc height ratio increased transiently in immediate postoperative period (from 0.18 to 0.21 ) and then, decreased significantly in last follow-up (0.18). In 'DIAM' group, disc height ratio increased transiently in immediate postoperative period (from 0.18 to 0.19 ), and then decreased significantly in the last follow-up (0.16). Three (16.7\%) and two $(28.6 \%)$ patients undergo on a re-operation due to severe back pain in 'Interspinous $U$ ' and 'DIAM' groups.

Conclusion: Long term follow up 'Interspinous U' and 'DIAM' group showed low patient satisfaction and poor radiological outcomes. To ascertain the benefit of PDS compare with posterior screw fixation, prospective analysis with larger population and multi-center study will be needed.

Key Words: Degenerative lumbar disease $\cdot$ Interspinous device $\cdot$ Prognosis

\section{INTRODUCTION}

As a surgical treatment for degenerative lumbar spinal stenosis, either simple decompression or spinal fusion has been performed so far ${ }^{12}$. However, simple decompression may cause instability, and spinal fusion which uses pedicle screws may need cause an excessive damage of soft tissues and bleeding, or cause complications such as pseudarthosis and adjacent segment disease ${ }^{4,10,13)}$. Therefore, posterior dynamic stabilizations (PDS) is attempted to overcome those problems ${ }^{14)}$. However,

- Received: May 4, 2012 - Revised: June 10, 2012

- Accepted: June 25, 2012

Corresponding Author: Sang Gu Lee, MD

Department of Neurosurgery, Gachon University Gil Hospital, 1198

Block, Guwol-dong, Namdong-gu, Incheon 405-760, Korea

Tel: +82-32-460-3304, Fax: +82-32-460-3899

E-mail: samddal@gilhospital.com the research about long-term prognosis of PDS is yet rare, and from some of the recent researches, a question arises about its prognosis and clinical efficacy ${ }^{11,16,17)}$. Therefore, this study intends to investigate whether this method can be replaced with the existing spinal fusion in the long run, by examining the long-term prognosis of PDS, especially 'Interspinous U' and 'DIAM'

\section{MATERIAL AND METHODS}

\section{Subjects}

As for the research subjects, this study selected patients who received single-level PDS with 'Interspinous U' (Spine motion, Germany) and 'DIAM' (Medtronic Ltd, Memphis, TN) and especially this study aimed at those whom a minimum of 60 months' follow-up observation could be conducted on.

As surgical subjects, this study selected patients who were 
diagnosed as degenerative spinal diseases including lumbar spinal stenosis and intervertebral disc herniation through a precise lumbar magnetic resonance imaging (MRI) and whose clinical symptoms were clear as spinal stenosis, such as neurogenic claudication, despite a sufficient period of conservative treatment.

Such medical instruments as 'Interspinous $U$ ' and 'DIAM' were used when patients were not diagnosed to need a pedicle screw fusion but more likely to have the secondary instability after both-side lumbar decompression or when a mild segmental instability was observed from the preoperative imaging study. Mild instabilities were not met Panjabi's ${ }^{9)}$ condition of spinal instability, but patients were selected when instability was dubitably found from flexion-extension lateral radiography.

\section{Surgical method}

As for surgeries for this study, a patient was made to take a prone position, and partial hemi-laminectomy and decompression were performed through the posterior approach, depending on the degree of stenosis. Besides, when intervertebral disc herniation was observed with nerve root compression accompanied, discectomy was performed. For patients into whom 'Interspinous $U$ ' was inserted, spinous process, supraspinous ligament and interspinous ligament were exposed in the area where the medical instrument was inserted, and the ligaments connected to it were removed. After 'Interspinous U' was inserted nearest to the dura mater by trimming the irregular cross section of spinous process, the wing part of 'Interspinous $U$ ' was compressed and fixed on the upper and lower spinous processes.

On the other hand, for patients into whom 'DIAM' was inserted, some space was secured between spinous processes and the size was measured while the supraspinous ligament was in the area for medical instrument insertion. Then, 'DIAM' was inserted, and a ligature was tied up between the upper and lower spinous processes and fixed steadily in place of suture.

\section{Analysis of patients' satisfaction}

The degrees of patients' postoperative satisfaction and functional recovery were analyzed according to such classifica- tions as Odom8), and patients were classified into 'Excellent, Good, Fair and Poor' (Table 1). Both the excellent and good grades were classified into 'Satisfactory Group', while both the fair and poor grades were classified into 'Unsatisfactory Group'.

\section{Radiological analysis}

To confirm the change of disc height ratio (DHR), this study performed a lateral lumbar X-ray projection on a regular basis to find out the height ratio between vertebral bodies. To minimize the variation of photographing, this study set up as a denominator the lateral length of the upper end plate of the lower vertebral body in the medical instrument-inserted segment, while setting up as a numerator the height of posterior disc, which is the length of a line connecting the posterior point of the lower end plate of the upper vertebral body to the posterior point of the upper end plate of the lower vertebral body in the medical instrument-inserted segment ${ }^{7}$. In the meantime, to find out the characteristics of 'Interspinous $U$ ',

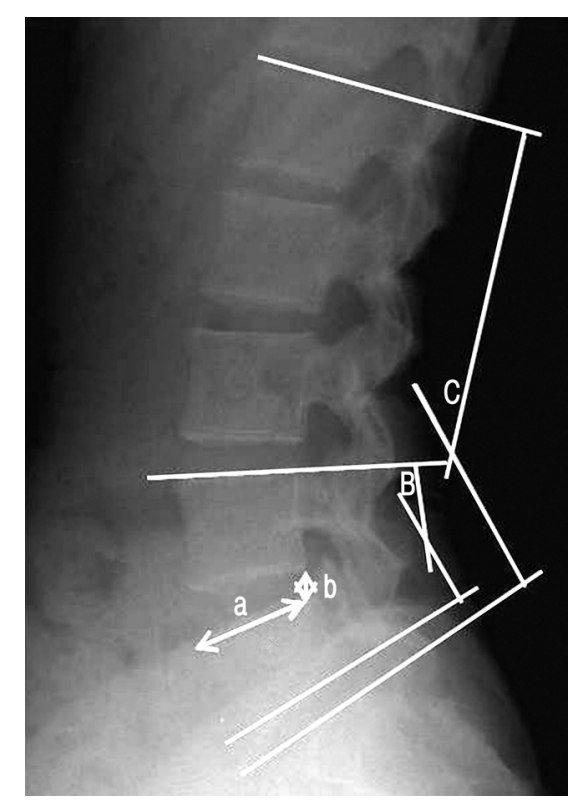

Fig. 1. Lateral plain X-ray of lumbar spine. $(a / b)$ : intervertebral disc height ratio (DHR), B: segmental lordotic angle (SLA), C: total lumbar lordotic angle (TLA)

Table 1. Odom's classification of patients' satisfaction

\begin{tabular}{ll}
\hline \hline & Indications \\
\hline Excellent & No symptoms and no difficulty in carrying out their daily and work lives \\
Good & Intermittent uncomfortable feelings but no difficulty in carrying out their work lives \\
Fair & Subjective symptoms were improved but had considerable difficulty in carrying out their daily activities \\
Poor & Symptoms were not improved at all or even worsened \\
\hline
\end{tabular}


which restricts extension while sustaining flexion, this study measured the total lumbar lordotic angle (TLA) and the segmental lordotic angle (SLA) where 'Interspinous $U$ ' was inserted. Finally, this study comparatively analyzed the change of DHR, TLA, and SLA before-and-after the surgery (Fig. 1). Comparison using the Wilcoxon signed rank test and $\mathrm{p}<0.05$ were considered significant statistically.

To investigate whether postoperative stability and bony erosion existed, this study performed a flexion and extension photographing a minimum of 12 months after the surgery and during the final follow-up period. In the X-ray photographing of flexion and extension, instability was determined when the sagittal plane displacement was $4.5 \mathrm{~mm}$ and the segment angle displacement exceeded 15 degrees at between L1 and L2, between L2 and L3 and between L3 and L4, or when it exceeded 20 degrees between lumbar vertebra L4 and L5 and 25 degrees between lumbar vertebra L5 and S1. Meanwhile, bony erosion of the spinous process was determined when the shade of the upper and lower spinous process was clearly reduced in the segment (where 'Interspinous $U$ ' or 'DIAM' was inserted) from the flexion and extension pictures.

\section{RESULTS}

There were total of 18 persons in the patient group with 'Interspinous $U$ ' inserted, and their average age was 58 (range, 35-73) years old, the gender ratio between male and female

Table 2. Demographic data of posterior dynamic stabilization patients

\begin{tabular}{lcc}
\hline \hline & Interspinous $U$ & DIAM \\
\hline Number of patients & 18 & 7 \\
Mean age (years) & 58.1 & 49.7 \\
(range) & $(35-73)$ & $(29-60)$ \\
Sex & & \\
$\quad$ Male & 8 & 7 \\
$\quad$ Female & 10 & 0 \\
Mean follow up (months) & 74.6 & 62.6 \\
(range) & $(60-88)$ & $(60-67)$ \\
\hline
\end{tabular}

was 8:10, and the average follow-up period was 74 (range, 60-88) months. There were 7 male in the patient group with 'DIAM' inserted, and their average age was 50 (range, 29-60) years old, and the average follow-up period was 62 (range, 60-67) months (Table 2).

\section{Analysis of patients' satisfaction}

In the investigation on patients' satisfaction performed during the final follow-up period, there were 2 patients in the excellent grade (11.1\%), 7 patients in the good grade (38.9\%) and $7(38.9 \%)$ and 2 patients (11.1\%) in the fair and poor grades respectively, out of the 'Interspinous U' group. Out of the 'DIAM' group, there were no one in the excellent grade, 3 patients $(42.9 \%)$ in the good grade, and $3(42.9 \%)$ and 1 patients (14.2\%) in the fair and poor grades respectively. It was found that the satisfactory group accounted for 9 patients (50.0\%) out of the 'Interspinous $U$ patient group and 3 patients (42.9\%) out of the 'DIAM' patient group (Table 3).

\section{Radiological analysis}

In the 'Interspinous U' patient group, the DHR from 0.18 \pm 0.03 to $0.21 \pm 0.03$ right after the surgery, but it decreased to $0.18 \pm 0.04$ at the final point of follow-up. Even in the 'DIAM' patient group, it increased from $0.18 \pm 0.06$ to $0.19 \pm 0.06$ right after the surgery, but it decreased to $0.16 \pm 0.03$ at the final follow-up.

Moreover, in the 'Interspinous U' patient group, the average TLA increased from $31.11 \pm 11.55$ degrees to $32.20 \pm 9.43$ degrees right after the surgery, further increasing more to 32.27

Table 3. The clinical outcome via Odom's criteria in the 'Interspinous U' group and 'DIAM' group at the last follow-up.

\begin{tabular}{lcc}
\hline & Interspinous $U$ & DIAM \\
\hline Excellent & 2 & 0 \\
Good & 7 & 3 \\
Fair & 7 & 3 \\
Poor & 2 & 1 \\
\hline
\end{tabular}

Table 4. Mean DHR, TLA and SLA of preoperative, postoperative and last follow-up X-rays

\begin{tabular}{|c|c|c|c|c|c|c|}
\hline & \multicolumn{3}{|c|}{ Interspinous U } & \multicolumn{3}{|c|}{ DIAM } \\
\hline & Preoperative & Postoperative & Last follow-up & Preoperative & Postoperative & Last followup \\
\hline DHR & $0.18 \pm 0.03$ & $0.21 \pm 0.03$ & $0.18 \pm 0.04$ & $0.18 \pm 0.06$ & $0.19 \pm 0.06$ & $0.16 \pm 0.03$ \\
\hline TLA & $31.11 \pm 11.55$ & $32.20 \pm 9.43$ & $32.27 \pm 7.27$ & $34.48 \pm 13.89$ & $33.95 \pm 7.96$ & $40.42 \pm 18.18$ \\
\hline SLA & $12.54 \pm 4.98$ & $9.23 \pm 4.23$ & $11.37 \pm 5.92$ & $15.17 \pm 4.68$ & $10.87 \pm 4.21$ & $14.81 \pm 6.30$ \\
\hline
\end{tabular}

DHR; disc height ratio, TLA; total lumbar lordotic angle, SLA; segmental lordotic angle 


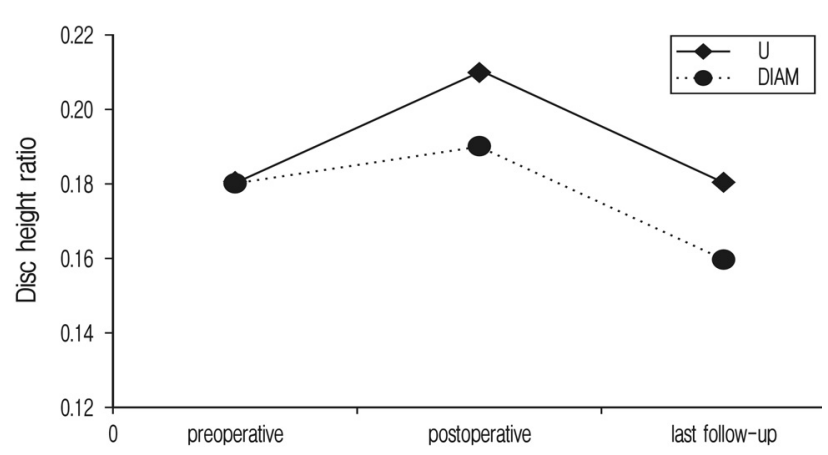

Fig. 2. Initially, Disk height ratio(DHR) was improved in both groups. However, it decreases in both group in minimum 5 year follow up in comparison to immediate postoperative value.

\pm 7.27 degrees at the final follow-up. In the 'DIAM' patient group, however, it decreased from $34.48 \pm 13.89$ degrees to $33.95 \pm 7.96$ degrees right after the surgery, but it was found to increase to $40.42 \pm 18.18$ degrees at the final follow-up.

The average SLA was $12.54 \pm 4.98$ degrees before the surgery in the 'Interspinous $U$ ' group, but it decreased to $9.23 \pm$ 4.23 degrees right after the surgery but increased to $11.37 \pm$ 5.92 degrees at the final point of follow-up. Even in the 'DIAM' group, it decreased from $15.17 \pm 4.68$ degrees to $10.87 \pm 4.21$ degrees right after the surgery, but it was found to increase to $14.81 \pm 6.30$ degrees at the final follow-up (Table 4 and Fig. 2). In both groups DHR, TLA, SLA changes statistically significant results did not indicate.

There were 3 cases of instability (16.7\%) in the 'Interspinous $U$ ' group, and 1 case (14.3\%) in the 'DIAM' group. Five patients (27.8\%) showed bony erosion in the 'Interspinous $U$ ' group, while 1 patient (14.3\%) was found in the 'DIAM' group (Fig. 3).

\section{Postoperative complications}

Due to aggravated symptom, there were 3 cases (16.7\%) found in the 'Interspinous U' group, which the medical instrument had to be removed and pedicle screw fusion had to be performed, and 2 cases found in the 'DIAM' group. There was no re-operation due to postoperative inflammation or hematoma in both the patient groups, and there was no cerebrospinal fluid leakage, medical instrument break-away or instrument fracture in both the patient groups neither.

\section{DISCUSSION}

To supplement the demerits of spinal fusion using pedicle screws and overcome 'Postoperative Lumbago Syndrome' resulted from damage in surrounding tissues during the surgery,

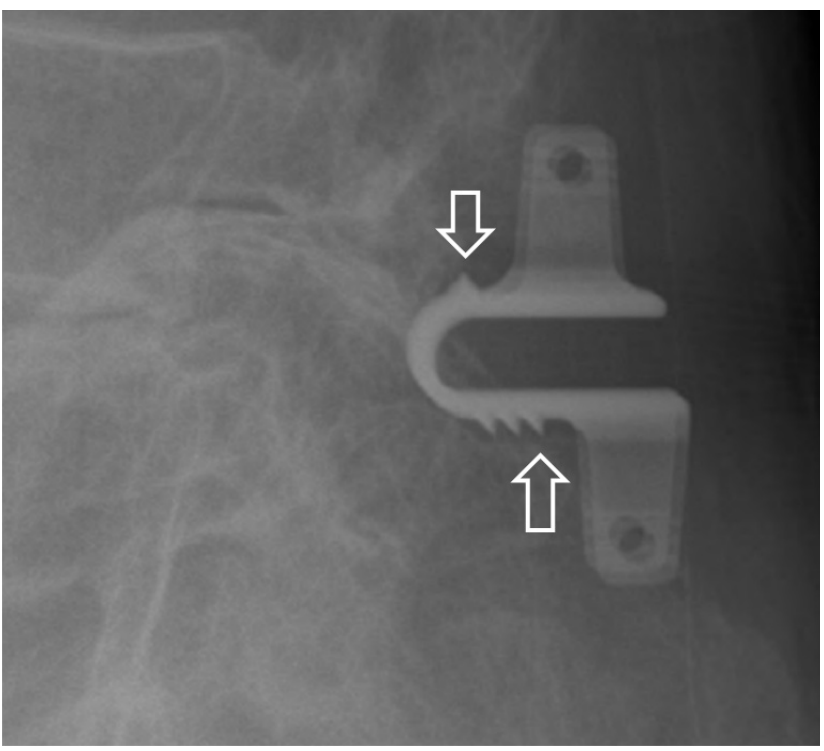

Fig. 3. Lateral radiography of female 73 years-old patient operated posterior dynamic stabilization on L4-5 level with 'Interspinous $U$ '. Marked bony erosion around interspinous $U$, especially around spikes, at 71 months follow-up, can be seen (arrows).

a new concept, called 'Dynamic Fusion' has been created. Its theoretical background is to preserve the spinal mobility of dynamic posterior fusion and to bring about the stabilization of more than one vertebral body segment ${ }^{3)}$. Those medical devices can be largely divided into three kinds; (1) posterior Interspinous device, (2) pedicle-based dynamic rod device, and (3) total facet replacement system ${ }^{6}$.

When it comes to patients' prognoses regarding PDS using interspinous devices, there were a small number of researches. According to a research conducted by Bono et $\mathrm{al}^{2}$. in 2007, $74 \%$ of the 106 patients showed unsatisfactory results. Adelt et $\mathrm{al}^{1)}$. carried out a multi-center study for 209 patients into whom 'Interspinous $U$ ' was inserted and a follow-up observation was conducted on for over 6 months (20 months on average), and it was found that about $89 \%$ of the patients showed satisfaction. In regard to the long-term prognoses of patients who received pedicle screw-based fusion, in comparison with researches on the PDS, Glaser et $\mathrm{al}^{5)}$. reported that $82 \%$ of the 94 patients, who had received pedicle screw-used fusion and been observed over 10 years, were found to be satisfied with their surgeries, and $69 \%$ of them showed a great degree of satisfaction, $81 \%$ had an intention to take the surgery again and $80 \%$ said they wanted to recommend this surgical method to others. In terms of patients' satisfaction according to the Odom's Classification, this study aimed at patients whom a minimum of 60 months follow-up observation could be conducted on. As a result, 9 patients (50.0\%) were in the satisfactory group respectively out of the 'Interspinous U' 
group. On the other hand, in the 'DIAM' group, there were 3 patients (42.9\%) in the satisfactory group respectively. In other words, it shows a low degree of patients' satisfaction, compared to the previous researches on PDS and pedicle screwused fusion.

As for prognoses of PDS radiologically analyzed, Park ea $\mathrm{al}^{11)}$ (2009). reported that the DHR decreased from 0.16 to 0.19 right after the surgery, further decreasing to 0.14 at the final follow-up, and $57 \%$ of the patients showed bony erosion which raised a question about its long-term efficiency. According to a research conducted by Yoon et $\mathrm{al}^{16)}$ (2008), the DHR increased from 0.19 to 0.23 right after the surgery but decreased to 0.17 at the final follow-up in the 'Interspinous $U$ ' patient group which had been observed for 39 months on average. On the other hand, in the 'DIAM' patient group which had been observed for 14 months on average, it changed to 0.17 to 0.19 right after the surgery but decreased to 0.18 at the final follow-up. Besides, there were a total of 5 cases (24\%) with bony erosion in the 'Interspinous $U$ ' group. Even in this study, the DHR was found to increase after the surgery but decrease during the final observation, and 5 patients (27.8\%) and 1 patient (14.3\%) were observed to have bony erosion in the 'Interspinous $U$ ' and 'DIAM' patients groups respectively. Since a decrease of intervals between vertebral bodies broadens the interval between the fixed posterior dynamic devices, ultimately expanding neural tubes, which seems to have insufficient effect in the long run. There were fewer cases of bony erosion found in this study. We assume that the reason was due to reduction of space between the inserted medical device and the spinous process as the interval between vertebral bodies decreased more than that of the long-term observation.

When it comes to the ratio of postoperative instability and re-operation, Yoon et $\mathrm{al}^{16}$. . reported that there were 3 (14.3\%) and 1 case $(6.25 \%)$ of mild instability found in the 'Interspinous $U$ ' and 'DIAM' patient groups respectively. Out of the 21 patients who had 'Interspinous $U$ ' inserted, 2 had 'Interspinous $U^{\prime}$ removed or received pedicle screw fusion. According to a research conducted by Park et $\mathrm{al}^{11)}$ in 2009 , out of the 31 patient who had 'Interspinous $U$ ' inserted, 1 (16.7\%) received re-operation. In this study, there were 3 cases (16.7\%) of postoperative instability found in the 'Interspinous U' patient group and 1 case (14.3\%) found in the 'DIAM' patient group. In the meantime, there were 3 cases (16.7\%) of re-operation in the 'Interspinous U' group and 2 cases (28.6\%) observed in the 'DIAM' group, which shows a negative result, compared to the previous researches on PDS, and shows a higher ratio than $12 \%$ of re-operation from the research on the longterm prognoses of patients on whom pedicle screw fusion was performed, conducted by Shin et $\mathrm{al}^{15)}$.
Based on all the findings such as patients' satisfaction, radiological analysis and the ratio of re-operation, it is doubtful about the long-term clinical utility of PDS by interspinous device, and it doesn't even show a favorable result, compared to other patients who had pedicle screw fusion performed. We think PDS with interspinous device have insufficient longterm effect widening interspinous space and foramens and maintain stability.

As for the limitations of this study, it is a retrospective study, and it was conducted in a single hospital with a small number of patients. Besides, since it aimed at patients on whom a long-term follow-up observation was carried out, a selective bias might be latent in this study. Therefore, further studies should be conducted with a large number of patients in more hospitals. If a prospective study can be carried out, by comparing a patient group with spinal fusion performed and the other group with simple decompression performed, it will be possible to exactly compare the prognosis and medical usefulness of PDS with interspinous device. In addition, the establishment of new indications for PDS will be needed.

\section{CONCLUSION}

Single-level PDS using 'Interspinous U' and 'DIAM' may have the merit of expanding the intervertebral foramen and preserving the spinal mobility, while taking advantages of performing a minimally-invasive surgery. However, as a result of the long-term follow-up observation, patients' satisfaction was not really high, with a high ratio of re-operation. This finding raises a question about the clinical efficacy of PDS. Therefore, in order to investigate the clinical usefulness of single-level PDS in the long run, it seems necessary to carry out a supplementary research in the near future.

\section{REFERENCES}

1. Adelt D, Samani J, Kim WK, Eif M, Lowery GL, Chomiak RJ: Coflex ${ }^{\circledR}$ interspinous stabilization: Clinical and radiographic results from an international multicenter retrospective study. Paradigm Spine Journal November 1:1-4, 2007

2. Bono CM, Vaccaro AR: Interspinous process devices in the lumbar spine. J Spinal Disord Tech 20:255-261, 2007

3. Christie SD, Song JK, Fessler RG: Dynamic interspinous process technology. Spine (Phila Pa 1976) 30:S73-S78, 2005

4. Deyo RA, Cherkin DC, Loeser JD, Bigos SJ, Ciol MA: Morbidity and mortality in association with operations on the lumbar spine. The influence of age, diagnosis, and procedure. J Bone Joint Surg Am 74:536-543, 1992

5. Glaser J, Stanley M, Sayre H, Woody J, Found E, Spratt K: A 10-year follow-up evaluation of lumbar spine fusion with 
pedicle screw fixation. Spine 28:1390-1395, 2003

6. Khoueir P, Kim KA, Wang MY: Classification of posterior dynamic stabilization devices. Neurosurg Focus 22:E3, 2007

7. Kong DS, Kim ES, Eoh W: One-year outcome evaluation after interspinous implantation for degenerative spinal stenosis with segmental instability. J Korean Med Sci 22:330-335, 2007

8. Odom GL, Finney W, Woodhall B: Cervical disc lesions. J Am Med Assoc 166:23-28, 1958

9. Panjabi MM: The stabilizing system of the spine. Part II. Neutral zone and instability hypothesis. J Spinal Disord 5:383-397, 1992

10. Park P, Garton HJ, Gala VC, Hoff JT, McGillicuddy JE: Adjacent segment disease after lumbar or lumbosacral fusion: review of the literature. Spine 29:1938-1944, 2004

11. Park SC, Yoon SH, Hong YP, Kim KJ, Chung SK, Kim HJ: Minimum 2-years follow-up result of degenerative spinal stenosis treated with interspinous $\mathrm{u}\left(\mathrm{Coflex}^{\mathrm{TM}}\right)$. J Korean Neurosurg Soc 46:292-299, 2009

12. Prolo DJ, Oklund SA, Butcher M: Toward uniformity in evaluating results of lumbar spine operations. A paradigm applied to posterior lumbar interbody fusions. Spine (Phila Pa 1976) 11: 601-606, 1986

13. Schlegel JD, Smith JA, Schleusener RL: Lumbar motion segment pathology adjacent to thoracolumbar, lumbar and lumbosacral fusions. Spine 21:971-981, 1996

14. Sengupta DK: Dynamic stabilization devices in the treatment of low back pain. Orthop Clin North AM 35:43-56, 2004

15. Shin BJ, Lee JC, Nam JS, Kim YB, Yoon SJ, Cho YI, et al: A minimum follow-up study of 10 years after pedicle screw fixation and fusion for degenerative lumbar diseases. Journal of Korean Spine Surg 13(1):1-9, 2006

16. Yoon SM, Lee SG, Kim EY, Kim WK: Follow-up comparison study of single-level posterior dynamic stabilization in lumbar degenerative disease 'Interspinous U' vs. 'DIAM'. Korean J Spine 5:136-141, 2008

17. Yoon SM, Lee SG, Park CW, Yoo CJ, Kim DY, Kim WK: Late complications of the single level 'interspinous $U$ ' in lumbar spinal stenosis with mild segmental instability. Korean J Spine 5:89-94, 2008 